\title{
Gear Fault Detection Based on Teager-Huang Transform
}

\author{
Hui Li, ${ }^{1}$ Haiqi Zheng, ${ }^{2}$ and Liwei Tang ${ }^{2}$ \\ ${ }^{1}$ Department of Electromechanical Engineering, Shijiazhuang Institute of Railway Technology, Shijiazhuang 050041, China \\ ${ }^{2}$ First Department, Shijiazhuang Mechanical Engineering College, Shijiazhuang 050003, China
}

Correspondence should be addressed to Hui Li, huili68@163.com

Received 17 August 2009; Accepted 12 February 2010

Academic Editor: Jerzy Sawicki

Copyright (C 2010 Hui Li et al. This is an open access article distributed under the Creative Commons Attribution License, which permits unrestricted use, distribution, and reproduction in any medium, provided the original work is properly cited.

\begin{abstract}
Gear fault detection based on Empirical Mode Decomposition (EMD) and Teager Kaiser Energy Operator (TKEO) technique is presented. This novel method is named as Teager-Huang transform (THT). EMD can adaptively decompose the vibration signal into a series of zero mean Intrinsic Mode Functions (IMFs). TKEO can track the instantaneous amplitude and instantaneous frequency of the Intrinsic Mode Functions at any instant. The experimental results provide effective evidence that Teager-Huang transform has better resolution than that of Hilbert-Huang transform. The Teager-Huang transform can effectively diagnose the fault of the gear, thus providing a viable processing tool for gearbox defect detection and diagnosis.
\end{abstract}

\section{Introduction}

Gears are important element in a variety of industrial applications such as machine tool and gearboxes $[1,2]$. An unexpected failure of the gear may cause significant economic losses. For that reason, fault diagnosis in gears has been the subject of intensive research. Vibration signal analysis has been widely used in the fault detection of rotation machinery. Many methods based on vibration signal analysis have been developed. These methods include power spectrum estimation, fast Fourier transform (FFT), cepstrum analysis, and envelope spectrum analysis, which have been proved to be effective in gear fault detection. However, these methods are based on the assumption of stationarity and linearity of the vibration signal. Therefore, new techniques are needed to analyze vibration signals for fault detection in gear system. Gear fault by their nature are time-localized transient events. To deal with nonstationary and nonlinear signals, timefrequency analysis techniques such as the Short-Time Fourier Transform (STFT) [1], Wavelet Transform (WT) [3-6], and Wigner-Ville distribution (WVD) [7-11] are widely used. The STFT [1] uses sliding windows in time to capture the frequency characteristics as functions of time. Therefore, spectrum is generated at discrete time instants. An inherent drawback with the STFT is the limitation between time and frequency resolutions. A finer frequency resolution can only be achieved at the expense of time resolution and vice versa. Furthermore, this method requires large amounts of computation and storage for display. The Wavelet Transform (WT) $[3,4]$ has been successfully used in nonstationary vibration signal processing and fault detection. WT is capable of providing both time-domain information and frequencydomain information simultaneously. A very appealing feature of the wavelet analysis is that it provides a uniform resolution for all the scales. Limited by the size of the basic wavelet function, the downside of the uniform resolution is uniformly poor resolution. Moreover, an important limitation of the wavelet analysis is its nonadaptive nature. Once the basic wavelet is selected, one will have to use it to analyze all the data. This leads to a subjective assumption on the characteristic of the analyzed signal. As a consequence, only signal features that correlate well with the shape of the wavelet function have a chance to lead to coefficients of high value. All other features will be masked or completely ignored. The Wigner-Ville distribution (WVD) [7-9] is a basic time-frequency representation, which is part of the Cohen class of distribution. Furthermore, it possesses a great number of good properties and is of popular interest for nonstationary signal analysis. Therefore, the Wigner-Ville distribution has received considerable attention in recent years as an analysis tool for nonstationary or time-varying signals. It has been widely used in the areas of structure-bone noise identification [12], optics [13], machinery condition monitoring [14-16], and so on. The difficulty with this 
method is the severe cross terms as indicated by the existence of negative power for some frequency ranges. In comparison, the Hilbert-Huang transform (HHT) is based on the instantaneous frequencies resulting from the intrinsic mode function of the being analyzed; thus, it is not constrained by the uncertainty limitations with respect to the time and frequency resolutions to which other timefrequency techniques are subject. In recent years, HHT has been applied to identification of damage time instant and location in biomedical signals processing [17-19], geophysics [20-23], image processing [24], structural testing [25], fault diagnosis [26], nuclear physics [27], and so on. These applications have further demonstrated the effectiveness of HHT in transient signal processing. The HHT estimates the instantaneous frequency (IF) using Hilbert transform (HT). However, owing to the inevitable window effect of HT, the demodulation results present noninstantaneous response characteristics. An alternative approach developed by Maragos et al. [28, 29], Vakman [30], Gexus and Boudraa $[31,32]$ uses a nonlinear energy tracking operator, Teager Kaiser energy operator (TKEO), to first estimate the energy required for generating an AM-FM signal and then separate it into its instantaneous frequency (IF) and instantaneous amplitude (IA) components. Note that the Hilbert transform approach mainly involves a linear integral operator, whereas the TKEO approach uses a nonlinear differential operator. TKEO gives a good estimate of IF and has low computational complexity [30-33].

In this paper, we present a new method to detect gear fault using empirical mode decomposition (EMD) and nonlinear Teager Kaiser Energy Operator (TKEO), which is named as Teager-Huang transform (THT) [32]. EMD is a fundamentally new approach to the decomposition of nonlinear and nonstationary signal presented originally by Huang et al. [34]. EMD can decompose multicomponent signals into a series of Intrinsic Mode Functions (IMFs), and then accurate instantaneous amplitude estimation can be acquired by TKEO. TKEO is a nonlinear operator, which has been developed by Teager. It can track the energy and identify the instantaneous frequency and instantaneous amplitude of monocomponent signal $[28,29,32]$. In the end, time-frequency spectrum is obtained by using TeagerHuang transform. The characteristic frequencies related to the gear defect can be effectively extracted. The basic method is introduced in detail. This approach is applied in the research of the fault detection and diagnosis of the gear wear. The experimental results show that this method can effectively monitor the gear fault.

To address the issues discussed above, this paper is organized as follows. Section 1 gives a brief introduction of the time-frequency analysis technology. Section 2 briefly describes the EMD and Hilbert-Huang transformation (HHT). Section 3 looks at the Teager-Huang transform (THT). Section 4 presents the method and procedure of the gear fault detection based on THT. Section 5 gives two simulation examples to show the effectiveness and reliability of the proposed method. Section 6 describes the experimental set-up. Section 7 gives the application of the method based on THT to fault detection of gear wear.
Finally, the main conclusions of this paper are provided in Section 8 .

\section{Introduction of Hilbert-Huang Transform}

Hilbert-Huang transformation is an emerging novel technique of signal decomposition having many interesting properties. In order to facilitate the reading of this paper we will introduce in detail the Hilbert-Huang transformation, which is a relatively novel technique.

2.1. The Concept of Intrinsic Mode Function. Huang et al. [34] have defined IMFs as a class of functions that satisfy two conditions

(1) in the whole data set, the number of extrema and the number of zero-crossings must be either equal or differ at most by one.

(2) at any point, the mean value of the envelope defined by the local maxima and the envelope defined by the local minima is zero.

2.2. Empirical Mode Decomposition (EMD). Empirical Mode Decomposition (EMD) has been proposed recently [20, 34] as an adaptive time-frequency data analysis method. It has proven to be quite versatile in a broad range of applications for extracting signals from data generated in noisy nonlinear and nonstationary processes $[28,29]$. As useful as EMD proved to be, it still leaves some annoying difficulties unresolved.

Empirical mode decomposition method is developed from the simple assumption that any signal consists of different simple intrinsic mode oscillations. The essence of the method is to identify the intrinsic oscillatory modes (IMFs) by their characteristic times scales in the signal and then decompose the signal accordingly. The characteristics time scale is defined by the time lapse between the successive extremes.

To extract the IMF from a given data set, the sifting process is implemented as follows. First, identify all the local extrema, and then connect all of the local maxima by a cubic spline line as the upper envelope. Then, repeat the procedure for the local minima to produce the lower envelope. The upper and lower envelopes should cover all the data between them. Their mean is designated $m_{1}(t)$, and the difference between the data and $m_{1}(t)$ is $h_{1}(t)$, that is,

$$
x(t)-m_{1}(t)=h_{1}(t) .
$$

Ideally, $h_{1}(t)$ should be an IMF, for the construction of $h_{1}(t)$ described above should have forced the result to satisfy all the definitions of an IMF by construction. To check if $h_{1}(t)$ is an IMF, we demand the following conditions: (i) $h_{1}(t)$ should be free of riding waves, that is, the first component should not display under-shots or over-shots riding on the data and producing local extremes without zero crossing; (ii) to display symmetry of the upper and lower envelops with respect to zero; and (iii) obviously the number of zero crossing and extremes should be the same in both functions. 
The sifting process has to be repeated as many times as it is required to reduce the extracted signal to an IMF. In the subsequent sifting process steps, $h_{1}(t)$ is treated as the data; then

$$
h_{1}(t)-m_{11}(t)=h_{11}(t),
$$

where $m_{11}(t)$ is the mean of the upper and lower envelops of $h_{1}(t)$.

This process can be repeated up to $k$ times; $h_{1 k}(t)$ is then given by

$$
h_{1(k-1)}(t)-m_{1 k}(t)=h_{1 k}(t) .
$$

After each processing step, checking must be done on whether the number of zero crossings equals the number of extrema.

The resulting time series is the first IMF, and then it is designated as $c_{1}(t)=h_{1 k}(t)$. The first IMF component from the data contains the highest oscillation frequencies found in the original data $x(t)$.

This first IMF is subtracted from the original data, and this difference is called a residue $r_{1}(t)$ by

$$
x(t)-c_{1}(t)=r_{1}(t) .
$$

The residue $r_{1}(t)$ is taken as if it was the original data and we apply to it again the sifting process. The process of finding more intrinsic modes $c_{i}(t)$ continues until the last mode is found. The final residue will be a constant or a monotonic function; in this last case it will be the general trend of the data:

$$
x(t)=\sum_{i=1}^{n} c_{i}(t)+r_{n}(t)
$$

Thus, one achieves a decomposition of the data into $n$ empirical IMF modes, plus a residue, $r_{n}(t)$, which can be either the mean trend or a constant.

2.3. The Hilbert-Huang Transform (HHT). Having obtained the IMFs using EMD method, one applies the Hilbert transform to each IMF component:

$$
H\left[c_{i}(t)\right]=\frac{1}{\pi} \int_{-\infty}^{+\infty} \frac{c_{i}(\tau)}{t-\tau} \mathrm{d} \tau .
$$

With this definition $c_{i}(t)$ and $H\left[c_{i}(t)\right]$ form a complex conjugate pair, which defines an analytic signal $z_{i}(t)$ :

$$
z_{i}(t)=c_{i}(t)+j H\left[c_{i}(t)\right],
$$

which can be expressed as

$$
z_{i}(t)=a_{i}(t) \exp \left(j \omega_{i}(t)\right) .
$$

With amplitude $a_{i}(t)$ and phase $\theta_{i}(t)$ defined by the expressions:

$$
\begin{aligned}
& a_{i}(t)=\sqrt{c_{i}^{2}(t)+H^{2}\left[c_{i}(t)\right]}, \\
& \theta_{i}(t)=\arctan \left(\frac{H\left[c_{i}(t)\right]}{c_{i}(t)}\right) .
\end{aligned}
$$
by

Therefore, the instantaneous frequency $\omega_{i}(t)$ can be given

$$
\omega_{i}(t)=\frac{\mathrm{d} \theta_{i}(t)}{\mathrm{d} t} .
$$
form:

Thus the original data can be expressed in the following

$$
x(t)=\operatorname{Re} \sum_{i=1}^{n} a_{i}(t) \exp \left(j \int \omega_{i}(t) d t\right),
$$

where the residue $r_{n}(t)$ has been left out. $\operatorname{Re}\{\cdot\}$ denotes the real part of a complex quantity.

Equation (11) enables us to represent the amplitude and the instantaneous frequency in a three-dimensional plot, in which the amplitude is the height in the time-frequency plane. This time-frequency distribution is designated as the Hilbert-Huang spectrum $H(\omega, t)$ :

$$
H(\omega, t)=\operatorname{Re} \sum_{i=1}^{n} a_{i}(t) \exp \left(j \int \omega_{i}(t) d t\right) .
$$

\section{Introduction of Teager-Huang Transform}

3.1. Teager Kaiser Energy Operator (TKEO). TKEO is a powerful nonlinear operator and has been successful used in many engineering application [31]. TKEO can track the modulation energy and identify the instantaneous frequency (IF) and the instantaneous amplitude (IA) of an amplitude modulation-frequency modulation (AM-FM) signal [29]. The TKEO, $\psi(\cdot)$ is defined for continuous-time signal $x(t)$ as follows $[31,32]$ :

$$
\psi[x(t)]=[\dot{x}(t)]^{2}-x(t) \ddot{x}(t),
$$

where $\dot{x}(t)$ and $\ddot{x}(t)$ are the first and the second time derivatives of $x(t)$, respectively. In the discrete case, the time derivatives may be approximated by time differences. In discrete-time domain, TKEO is given as follows [28, 32]:

$$
\psi[x(n)]=x^{2}(n)-x(n+1) \cdot x(n-1) .
$$

The instantaneous frequency $f(n)$ and instantaneous amplitude $|a(n)|$ at any time instant of the AM-FM signal $x(n)$ can, respectively, be given as follows [28]:

$$
\begin{gathered}
y(n)=x(n)-x(n-1), \\
f(n)=\arccos \left(1-\frac{\psi[y(n)]+\psi[y(n+1)]}{4 \psi[x(n)]}\right), \\
|a(n)|=\sqrt{\frac{\psi[x(n)]}{\sin ^{2}[f(n)]}}, \\
f(n)=\frac{1}{2} \arccos \left(1-\frac{\psi[x(n+1)-x(n-1)]}{2 \psi[x(n)]}\right), \\
|a(n)|=\frac{2 \psi[x(n)]}{\sqrt{\psi[x(n+1)-x(n-1)]}} .
\end{gathered}
$$




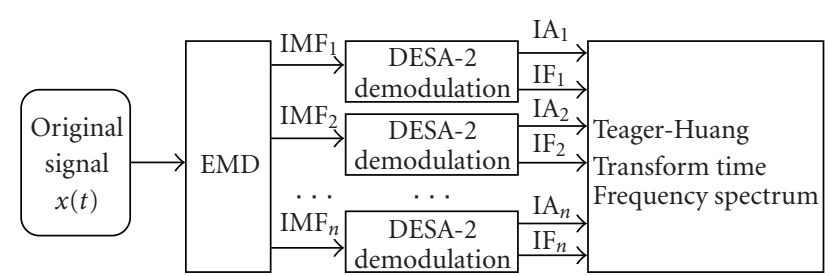

Figure 1: The flow chart of Teager-Huang Transform.

In general, the demodulation method given by (15), (16), and (17) is named as discrete energy separation algorithm-1 (DESA-1). The demodulation method given by (18) and (19) is called as discrete energy separation algorithm-2 (DESA-2) [35]. In this paper, we calculate the instantaneous frequency $f(n)$ and instantaneous amplitude $|a(n)|$ by DESA-2. The DESA-2 algorithm only requires three samples for the energy computation at each time instant and is less computationally complex. Therefore, the DESA-2 algorithm has an excellent time resolution and almost instantanoeus. This excellent time resolution provides us with the ability to capture the energy fluctuations of the AM-FM signal.

3.2. The Teager-Huang Transform. In order to estimate the instantaneous frequency $f(n)$ and instantaneous amplitude $|a(n)|$ of $x(t)$, the EMD is combined with the TKEO. TKEO can only be used to track the IF and IA of a monocomponent AM-FM signal. If $x(t)$ is a multicomponent AM-FM signal, then bandpass filtering is needed to isolate each component before applying the discrete energy separation algorithm (DESA). Therefore, the EMD is used as a multiband filtering to separate the signal components in the time domain and hence reduce multicomponent demodulation to multicomponent one. The association of the EMD and the TKEO methods is called as Teager-Huang transform (THT) [32].

According to (18) and (19) the original data can be expressed in the following form:

$$
x(t)=\sum_{i=1}^{n}\left|a_{i}(t)\right| \exp \left(j \int 2 \pi f_{i}(t) d t\right) .
$$

Equation (20) enables us to represent the amplitude and the instantaneous frequency in a three-dimensional plot, in which the amplitude is the height in the time-frequency plane. This time-frequency distribution is designated as the Teager-Huang spectrum $T(f, t)$ :

$$
T(f, t)=\sum_{i=1}^{n}\left|a_{i}(t)\right| \exp \left(j \int 2 \pi f_{i}(t) d t\right) .
$$

The final presentation of the the IF and the IA results is an energy time frequency representation. The block diagram of Teager-Huang transform technique is illustrated in Figure 1. The original vibration signal can be decomposed into a series of monocomponent AM-FM signal named as IMFs. Then the IF and IA of the separated IMFs are calculated using the DESA-2 algorithm.

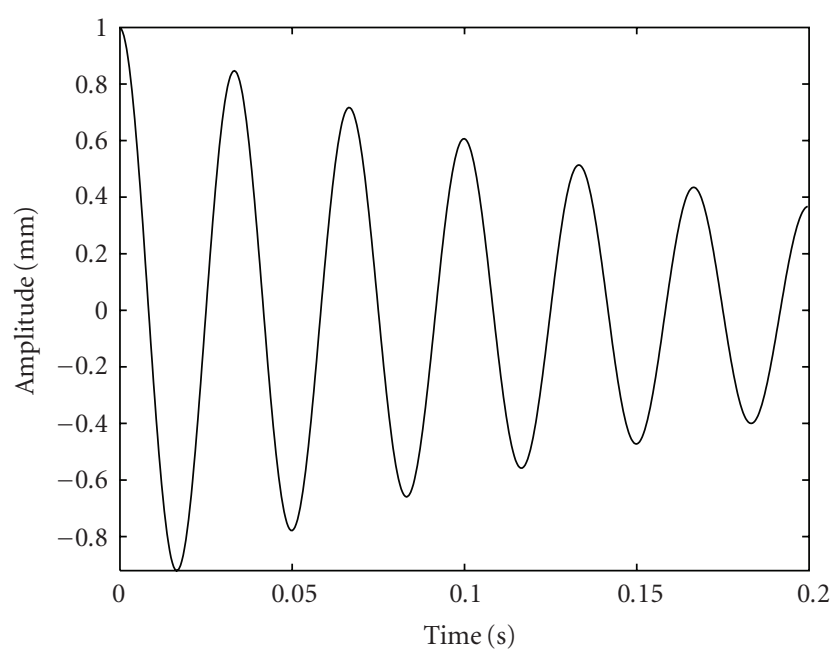

FIgURE 2: The monocomponent signal.

\section{Proposed THT Method to Fault Detection and Diagnosis of Gear}

The procedure of proposed THT spectrum method is given as follows:

(1) to decompose the vibration signal $x(t)$ using EMD and to obtain IMFs,

(2) to calculate the THT spectrum according to Section 3,

(3) to draw a diagnostic conclusion according to the THT spectrum.

\section{Signal Simulation of THT Spectrum}

The performance of the proposed method has been assessed by means of tests on two simulative signals. Several monocomponent and multicomponents signals, characterized by known instantaneous frequency trajectories, have been considered. The evolution versus time of the instantaneous frequency and amplitude of each component of the analyzed signal is finally shown.

5.1. Tests on Monocomponent Signals. The main objective of these tests is to establish the measurement accuracy of the proposed method as well as its advantages in IF and IA estimation. As an example, Figure 2 gives a monocomponent signal whose analytic expression in the time domain is

$$
x(t)=a(t) \cdot s(t),
$$

where $a(t)$ and $s(t)$ are given as follows:

$$
\begin{gathered}
a(t)=e^{-5 t}, \\
s(t)=\cos (60 \pi t) .
\end{gathered}
$$

The number of acquired samples is equal to 512 and the sampling frequency is $1920 \mathrm{~Hz}$. Figure 3 displays the THT spectrum estimated by the proposed estimation method. 


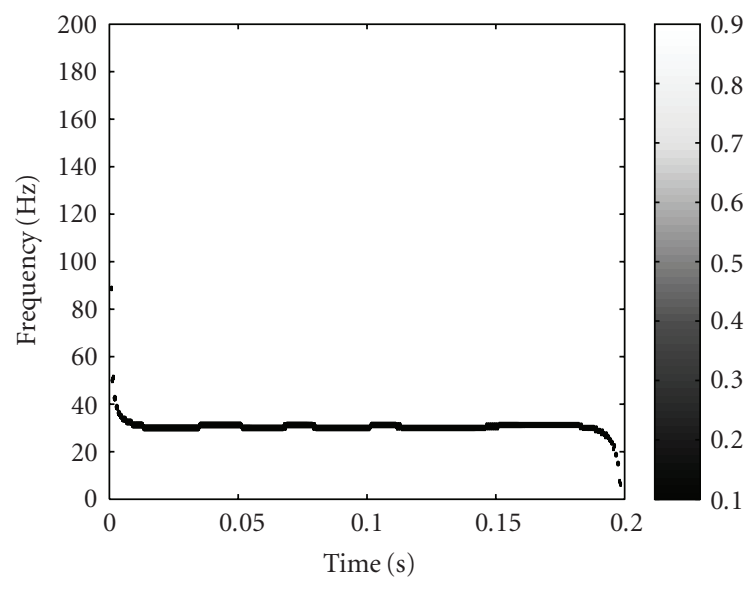

FIGURE 3: THT spectrum of monocomponent signal.

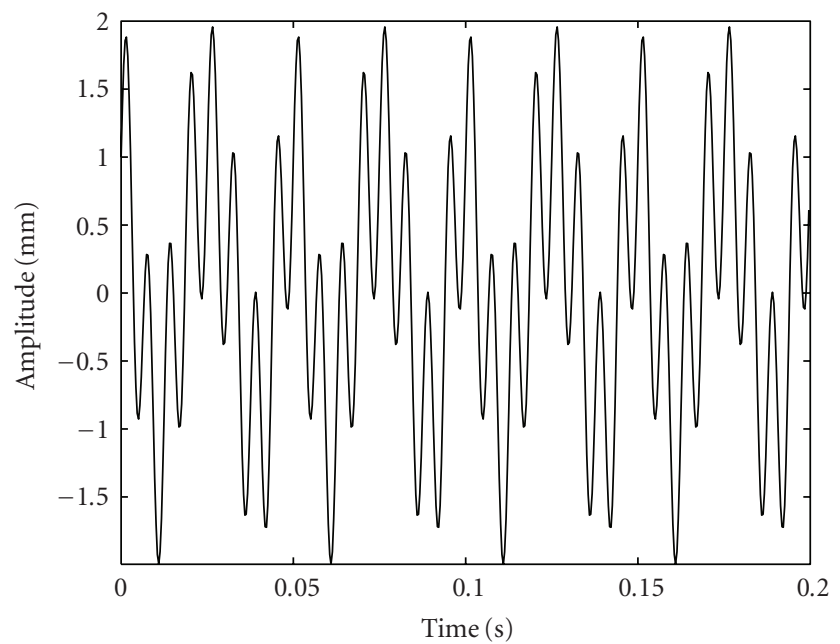

Figure 4: The multicomponent signal.

5.2. Tests on Multicomponent Signals. A significant example is shown in Figure 4. The signal shown in Figure 4 is composed of two components according to

$$
\begin{gathered}
x_{1}(t)=\cos [2 \pi 40 t+0.5 \sin (2 \pi 20 t)], \\
x_{2}(t)=\sin (2 \pi 160 t), \\
x(t)=x_{1}(t)+x_{2}(t) .
\end{gathered}
$$

Signal $x(t)$ is composed of a carrier frequency of $40 \mathrm{~Hz}$, frequency-modulated of $20 \mathrm{~Hz}$, and constant or timeindependent frequency of $160 \mathrm{~Hz}$ sine wave.

Therefore, the frequency-modulated $f(t)$ can be written as

$$
f(t)=40+10 \cos (40 \pi t) .
$$

The variation range of the frequency-modulated $f(t)$ is given as

$$
30 \leq f(t) \leq 50 .
$$
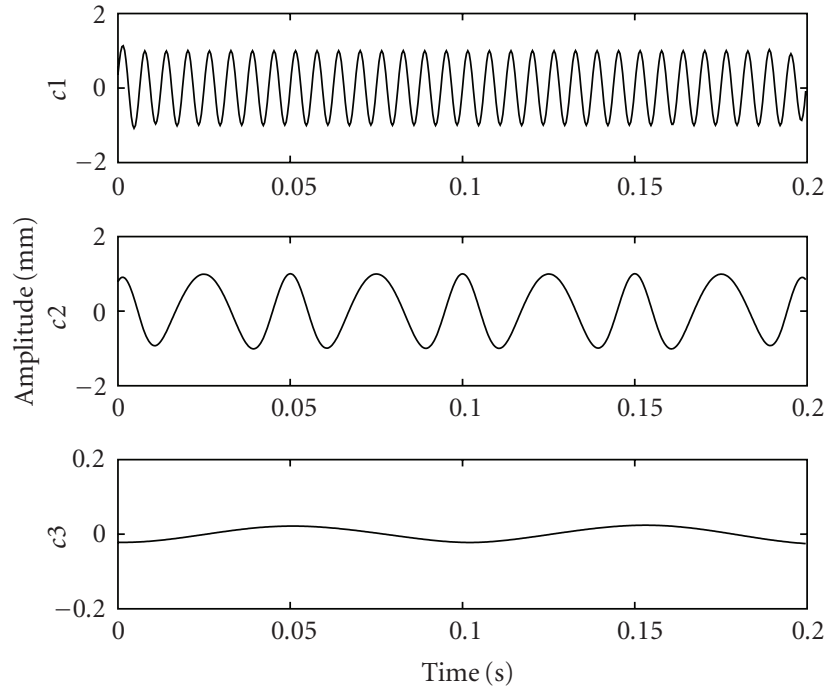

Figure 5: The three IMFs component.

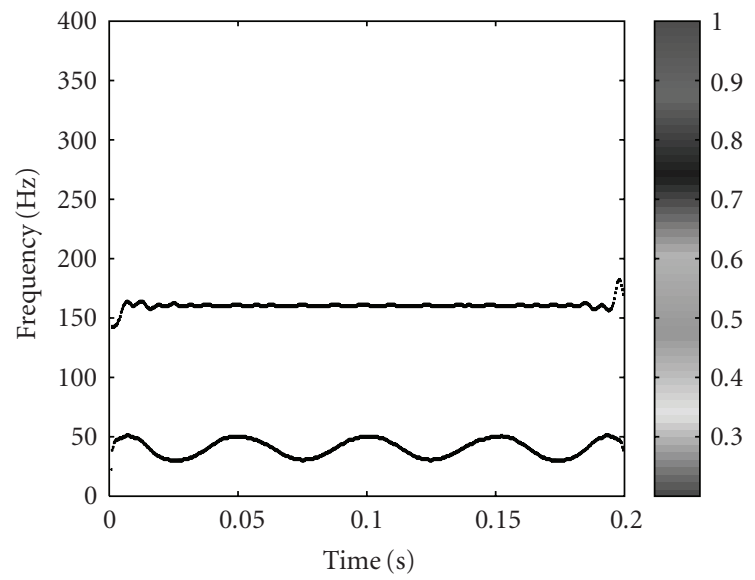

FIgURE 6: THT spectrum of multicomponent signal

Figure 4 displays a graphical sketch of the signal $x(t)$, which was generated over a total time span $T=0.2 \mathrm{~s}$ with a sampling frequency $f_{s}=2560 \mathrm{~Hz}$. Figure 5 shows the empirical mode decomposition in IMFs of the signal $x(t)$. With the help of the sifting algorithm explained in Section 2, we carried out this decomposition. The decomposition identifies two modes: $c_{1}$ represents the sine wave of $x_{2}(t)$, $c_{2}$ represents the frequency modulated signal, $x_{1}(t)$, and $c_{3}$ is the residue, respectively. By virtue of EMD method, signal can be decomposed into two complete and orthogonal intrinsic mode functions. Therefore, we can know not only the frequency components of the signal but also the variation of the amplitude and period. These IMFs component can reflect the actual physical meaning of the signal.

Figure 6 displays the instantaneous frequency and the instantaneous amplitude estimation of multicomponent signal $x(t)$ based on the empirical mode decomposition and TKEO. The IF in Figure 6 shows a clear picture of temporal frequency distribution of the data; that is, the linear response has constant frequency at $160 \mathrm{~Hz}$, and the nonlinear response has frequency dependence modulated around $40 \mathrm{~Hz}$ and 


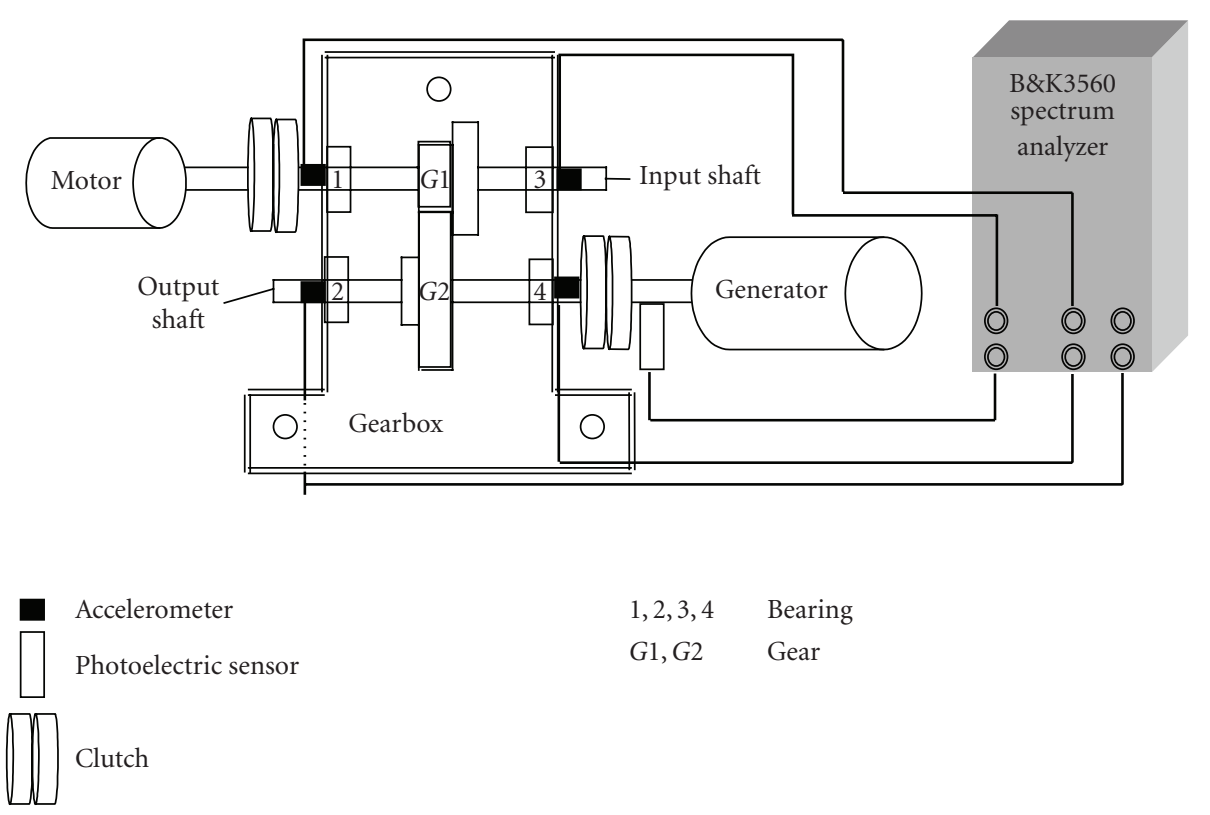

FIgURE 7: Experimental set-up.

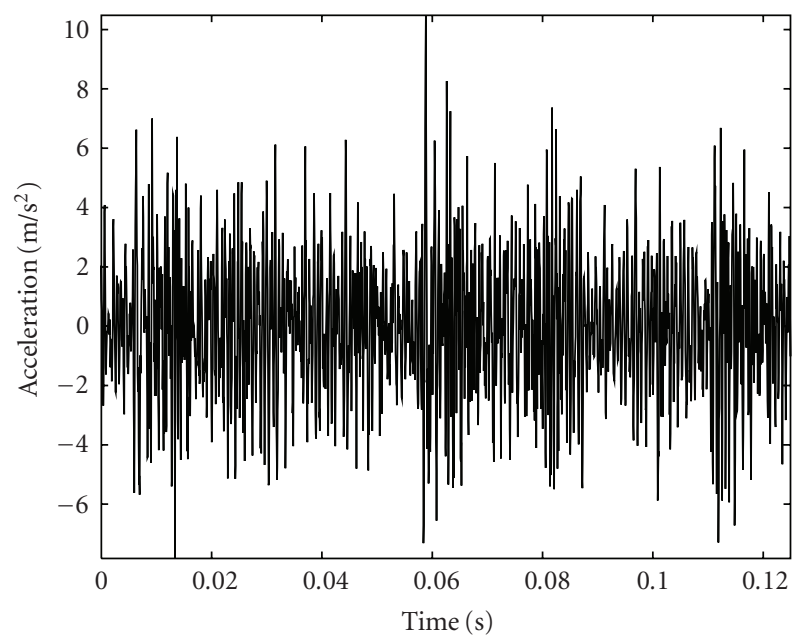

Figure 8: Time-domain vibration signal with gear wear.

bounded by 30 and $50 \mathrm{~Hz}$, and the decaying energy of the nonlinear response with the color changing from the white at the beginning to dark blue at the end of the record. Therefore, the instantaneous frequency and the instantaneous amplitude estimation based on the empirical mode decomposition and TKEO is better to describe the characteristics of the time-frequency distribution.

These simple simulation examples illustrate the effectiveness of the THT spectrum for analyzing transient (or nonstationary) vibration signal. The results demonstrate that THT is suited for capturing transient events in dynamic system. THT provides a viable signal processing tool for machine fault detection and diagnosis.

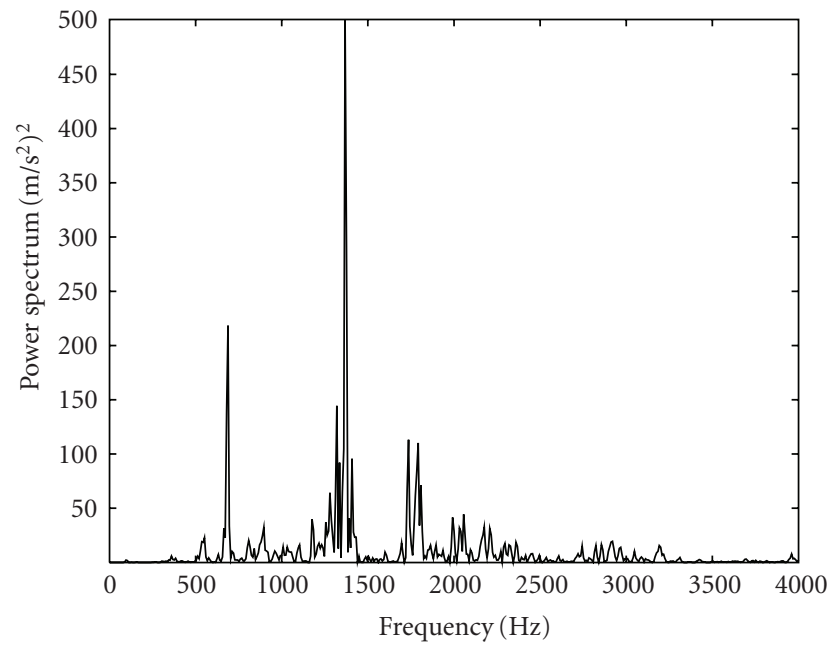

Figure 9: Power spectrum of the vibration signal.

\section{Experimental Set-Up}

A crack, wear, or broken gear tooth failure may cause fatal accidents; so the recognition of gear tooth fault is very important for the safety of a gearbox. The experimental setup consists of a single-stage gearbox, driven by a $4.5 \mathrm{~kW}$ AC governor motor. The driving gear has 28 teeth and the driven gear has 36 teeth. Therefore, the transmission ratio is $36 / 28$, which means that a decrease in rotation speed is achieved. The module of the gear is $2.5 \mathrm{~mm}$. Localized wear defect of the driven gear had a chipped tooth, from zero thickness at pitch point to $25 \%$ thichness at the tooth top, to simulate serious wear. The input speed of the spindle was 


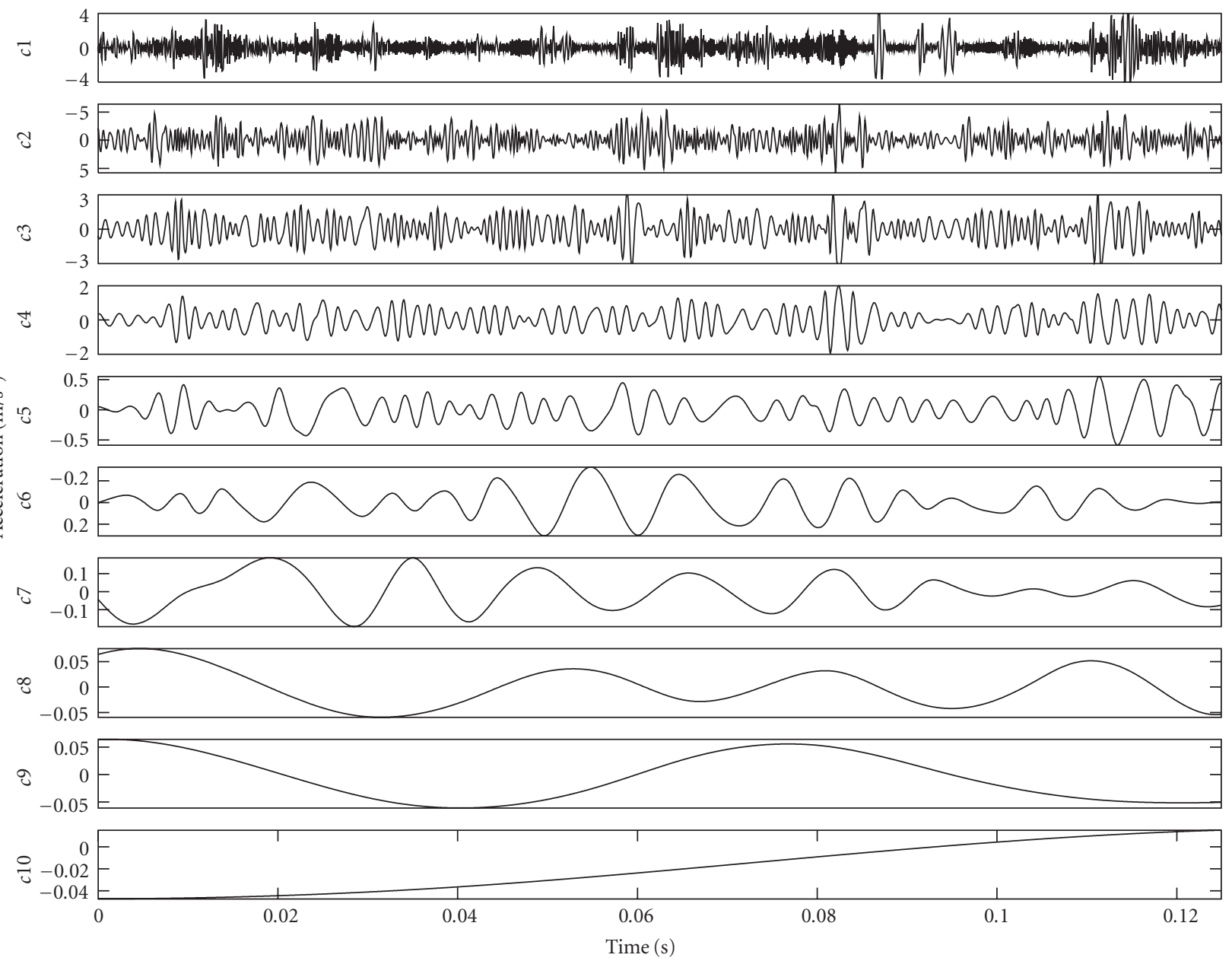

FIgURE 10: IMFs of the signal shown in Figure 8.

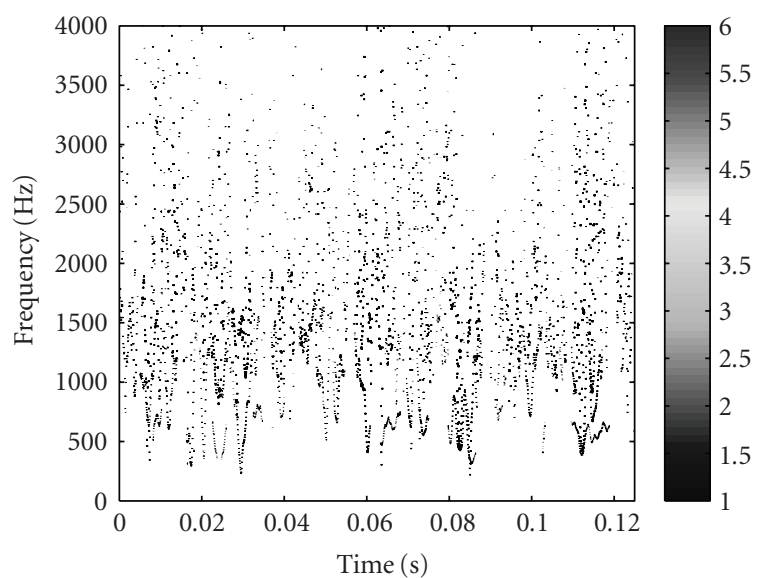

Figure 11: HHT spectrum of vibration signal shown in Figure 8.

$1473 \mathrm{r} / \mathrm{min}$; that is, the rotating frequency of the output shaft $f_{r}$ was $19.11 \mathrm{~Hz}$. Therefore, the tooth-meshing frequency was $688 \mathrm{~Hz}$. The monitoring and diagnostic system was composed of four accelerometers, amplifiers, B\&K 3560 spectrum analyzer, and a computer. The sampling span was $6.4 \mathrm{kHz}$, the sampling frequency was $16384 \mathrm{~Hz}$, and the sampling points were 2048. After sampling, the measured

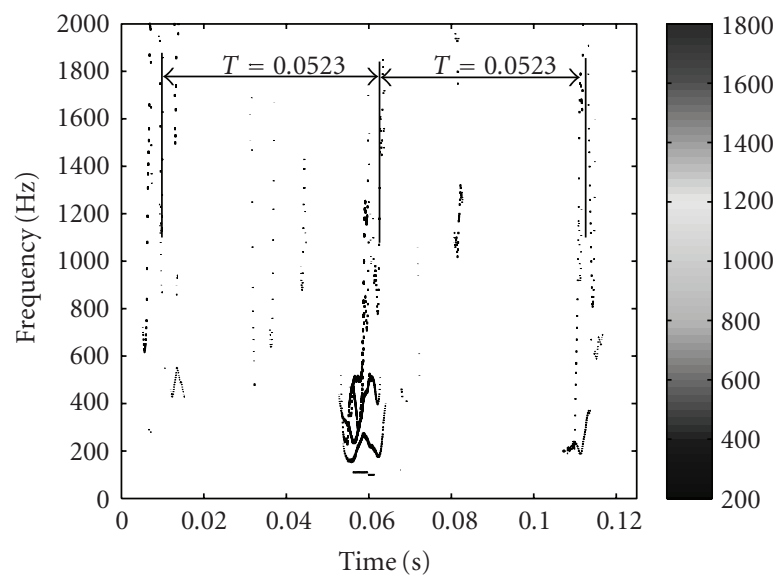

FIgURE 12: THT spectrum of vibration signal shown in Figure 8.

vibration signals were loaded into MATLAB from datafiles.

\section{Gear Fault Detection and Diagnosis Based on THT}

It is well known that the most important components in gear vibration spectra are the tooth-meshing frequency and 
its harmonics, together with sidebands due to modulation phenomena. The increment in the number and amplitude of such sidebands may indicate a fault condition. Moreover, the spacing of the sidebands is related to their source. In particular, fault localized on one tooth or a few teeth, such as gear crack or gear wear, produces modulation effects only during the engagement of the faulted teeth, but repeated once each revolution of the gear. As a consequence, the spectrum presents a large number of sidebands of the tooth-meshing frequency and its harmonics, spread over a wide frequency range, spaced by the rotation frequency of the faulted gear, and characterized by low amplitude [27].

The original vibration signal with gear wear fault is displayed in Figure 8. It is clear that there are periodic impacts in the vibration signal. There are significant fluctuations in the peak amplitude of the signal. However, it is hardly possible to evaluate the gear fault condition only through such time domain vibration signal.

Figure 9 shows the power spectrum of the vibration signal with gear wear. $688 \mathrm{~Hz}$ and $1376 \mathrm{~Hz}$ frequency component, which are the first and second harmonic of the meshing frequency, can be clearly seen in Figure 9. But there are no fault frequency components around $19.11 \mathrm{~Hz}$. Therefore, classical Fourier analysis has some limitation such as unable to process nonstationary signals.

To the data of Figure 8, the EMD algorithm is applied. Figure 10 displays the empirical mode decomposition in ten IMFs of the vibration signal in Figure 8. The decomposition identifies ten modes: $c_{1} \sim c_{9}$ represents the frequency components excited by the gear wear defects, and $c_{10}$ is the residue, respectively. Mode $c_{1}$ contains the highest signal frequencies, mode $c_{2}$ the next higher-frequency band, and so on.

From Figure 10, it can be easily proven that the EMD decomposes vibration signal very effectively on an adaptive method. The Hilbert-Huang transform (HHT) can be applied to each IMF $c_{i}(t)$, resulting in HHT spectrum according to [27]. The corresponding results of the HHT analysis are illustrated in Figure 11. From Figure 11, it is clearly seen that the vibration energy is mainly concentrated on tooth-meshing frequency $(688 \mathrm{~Hz})$ and its harmonics, in which their amplitudes are higher than others. The energy distracts from $500 \mathrm{~Hz}$ to $3500 \mathrm{~Hz}$. The HHT spectrum displays no repetitive signal patterns, without providing specific information on gear defect propagation. In comparison, the corresponding results of the THT analysis are illustrated in Figure 12. The presence of gear wear fault results in a sudden increase of vibration energy. In Figure 12, the instantaneous amplitude is relatively high and has the period impulse associated with period $(0.0523 \mathrm{~s})$ of the output shaft revolution, which corresponds to a $19.11 \mathrm{~Hz}$ repetitive frequency resulting from the structural defect on the gear of output shaft. Such repetitive frequency reflects degradation of the gear health condition as the defect propagated through the gear meshing. Physically, impacts generated by the gear meshing excite intrinsic modes of the gear system, giving rise to a train of transient vibrations. To compare Figure 11 with Figure 12, it is easily concluded that the THT has better resolution than that of HHT. Thus, the THT has shown to provide an effective tool for gear fault detection and diagnosis.

\section{Conclusions}

A method for fault diagnosis of gear wear was presented based on a newly developed signal processing technique named as empirical mode decomposition (EMD) and Teager Kaiser Energy Operator (TKEO). Using EMD method, the original vibration signals of gear fault can be decomposed into intrinsic modes. Therefore, we can recognize the vibration modes that coexist in the system, and to have a better understanding of the nature of the fault information contained in the vibration signal. According to Teager-Huang transform spectrum, the characteristic period of the gear fault can be easily recognized. Practical vibration signal monitored from a gearbox with gear fault is analyzed by the presented method. The experimental result has been shown that Teager-Huang transform can be used as an effective diagnostic method for gear fault. Teager-Huang transform has better resolution than Hilbert-Huang transform. Such a technique can be further applied to the health detection of other of dynamic systems, such as electrical drives. Research is being continued to systematically investigate the suitability and constraints of the THT for nonstationary signal analysis, using vibration signals from different fault types of gear.

\section{Acknowledgments}

The authors are grateful to the National Natural Science Foundation of China (no. 50775219 and no. 50975185), and Zhejiang Provincial Natural Science Foundation (no. Y1080040). The authors are also grateful to the editors and anonymous reviewers for their constructive comments.

\section{References}

[1] L. Cohen, Time-Frequency Analysis, Prentice-Hall, Englewood Cliffs, NJ, USA, 1995.

[2] J. Lin and L. Qu, "Feature extraction based on morlet wavelet and its application for mechanical fault diagnosis," Journal of Sound and Vibration, vol. 234, no. 1, pp. 135-148, 2000.

[3] W. J. Staszewski, "Wavelet based compression and feature selection for vibration analysis," Journal of Sound and Vibration, vol. 211, no. 5, pp. 735-760, 1998.

[4] C. J. Li and J. Ma, "Wavelet decomposition of vibrations for detection of bearing-localized defects," NDT \& E International, vol. 30, no. 3, pp. 143-149, 1997.

[5] S. Prabhakar, A. R. Mohanty, and A. Sekhar, "Application of discrete wavelet transform for detection of ball bearing race faults," Tribology International, vol. 35, no. 12, pp. 793-800, 2002.

[6] W. J. Wang and P. D. McFadden, "Application of orthogonal wavelets to early gear damage detection," Mechanical Systems and Signal Processing, vol. 9, no. 5, pp. 497-507, 1995.

[7] W. J. Staszewski, K. Worden, and G. R. Tomlinson, "Thefrequency analysis in gearbox fault detection using the Wigner-Ville distribution and pattern recognition," Mechanical Systems and Signal Processing, vol. 11, no. 5, pp. 673-692, 1997.

[8] L. Hui, Z. Haiqi, and T. Liwei, "Wigner-Ville distribution based on EMD for faults diagnosis of bearing," Fuzzy Systems and Knowledge Discovery, vol. 4223, pp. 803-812, 2006. 
[9] G. Matz and F. Hlawatsch, "Wigner distribution (nearly) everywhere: time-frequency analysis of signals, systems, random process, signal spaces, and frames," Signal Processing, vol. 83, no. 7, pp. 1355-1378, 2003.

[10] B. Boashash, Time-Frequency Signal Analysis and Processing, Prentice-Hall, Englewood Cliffs, NJ, USA, 2003.

[11] F. Hlawatsch and W. Kozek, "Wigner distribution of a linear signal space," IEEE Transactions on Signal Processing, vol. 41, no. 3, pp. 1248-1258, 1993.

[12] T. J. Wahl and J. S. Bolton, "The application of the Wigner distribution to the identification of structure-borne noise components," Journal of Sound and Vibration, vol. 163, no. 1, pp. 101-122, 1993.

[13] H. O. Bartelt, K.-H. Brenner, and A. W. Lohmann, "The Wigner distribution function and its optical production," Optics Communications, vol. 32, no. 1, pp. 32-38, 1980.

[14] Q. Meng and L. Qu, "Rotating machinery fault diagnosis using Wigner distribution," Mechanical Systems and Signal Processing, vol. 5, no. 3, pp. 155-166, 1991.

[15] J. Leuridan and H. V. D. Auweraer, "The analysis of nonstationary dynamics signals," Sound and Vibration, vol. 11, pp. 14-26, 1994.

[16] Y. S. Shin and J. J. Jeon, "Pseudo Wigner-Ville time-frequency distribution and its application to machinery condition monitoring," Shock and Vibration, vol. 1, pp. 65-76, 1993.

[17] W. Huang, Z. Shen, N. E. Huang, and Y. C. Fung, "Nonlinear indicial response of complex nonstationary oscillations as pulmonary hypertension responding to step hypoxia," Proceedings of the National Academy of Sciences of the United States of America, vol. 96, no. 5, pp. 1834-1839, 1999.

[18] W. Huang, Z. Shen, N. E. Huang, and Y. C. Fung, "Engineering analysis of biological variables: an example of blood pressure over 1 day," Proceedings of the National Academy of Sciences of the United States of America, vol. 95, no. 9, pp. 4816-4821, 1998.

[19] W. Huang, Z. Shen, N. E. Huang, and Y. C. Fung, "Use of intrinsic modes in biology: examples of indicial response of pulmonary blood pressure to step hypoxia," Proceedings of the National Academy of Sciences of the United States of America, vol. 95, no. 22, pp. 12766-12771, 1998.

[20] N. E. Huang, Z. Shen, and S. R. Long, "A new view of nonlinear water waves: the Hilbert spectrum," Annual Review of Fluid Mechanics, vol. 31, pp. 417-457, 1999.

[21] L. Wang, C. Koblinsky, S. Howden, and N. Huang, "Interannual variability in the South China Sea from expendable bathythermograph data," Journal of Geophysical Research, vol. 104, no. 10, pp. 23509-23523, 1999.

[22] M. L. C. Wu, S. Schubert, and N. E. Huang, "The development of the South Asian summer monsoon and the Intraseasonal oscillation," Journal of Climate, vol. 12, no. 7, pp. 2054-2075, 1999.

[23] M. Dätig and T. Schlurmann, "Performance and limitations of the Hilbert-Huang transformation (HHT) with an application to irregular water waves," Ocean Engineering, vol. 31, no. 1415, pp. 1783-1834, 2004.

[24] J. C. Nunes, Y. Bouaoune, E. Delechelle, O. Niang, and P. Bunel, "Image analysis by bidimensional empirical mode decomposition," Image and Vision Computing, vol. 21, no. 12, pp. 1019-1026, 2003.

[25] S. T. Quek, P. S. Tua, and Q. Wang, "Detecting anomalies in beams and plate based on the Hilbert-Huang transform of real signals," Smart Materials and Structures, vol. 12, no. 3, pp. 447460, 2003.
[26] H. Li, Y. Zhang, and H. Zheng, "Hilbert-Huang transform and marginal spectrum for detection and diagnosis of localized defects in roller bearings," Journal of Mechanical Science and Technology, vol. 23, no. 1, pp. 291-301, 2009.

[27] L. Hui, Z. Yuping, and Z. Haiqi, "Wear detection in gear system using Hilbert-Huang transform," Journal of Mechanical Science and Technology, vol. 20, no. 11, pp. 1781-1789, 2006.

[28] P. Maragos, J. F. Kaiser, and T. F. Quatieri, "Energy separation in signal modulations with application to speech analysis," IEEE Transactions on Signal Processing, vol. 41, no. 10, pp. 3024-3051, 1993.

[29] P. Maragos, J. F. Kaiser, and T. F. Quatieri, "On amplitude and frequency demodulation using energy operators," IEEE Transactions on Signal Processing, vol. 41, no. 4, pp. 1532-1550, 1993.

[30] D. Vakman, "On the analytic signal the Teager-Kaiser energy algorithm and other methods for defining amplitude and frequency," IEEE Transactions on Signal Processing, vol. 44, no. 4, pp. 791-797, 1996.

[31] J. C. Cexus and A. O. Boudraa, "Teager-Huang analysis applied to sonar target recognition," International Journal of Signal Processing, vol. 1, no. 1, pp. 23-27, 2004.

[32] J. C. Cexus and A. O. Boudraa, "Nonstationary signals analysis by Teager-Huang Transform (THT)," in Proceedings of the 13th European Signal Processing Conference (EUSIPCO '06), pp. 1$5,2006$.

[33] A. Potamianos and P. Maragos, "A comparisom of the energy operator and the Hilbert transform approach to signal and speech demodulation," Signal Processing, vol. 37, no. 1, pp. 95120, 1994.

[34] N. E. Huang, Z. Shen, S. R. Long, et al., "The empirical mode decomposition and the Hilbert spectrum for nonlinear and non-stationary time series analysis," Proceedings of Royal Society London, A, vol. 454, no. 1971, pp. 903-995, 1998.

[35] H. Li, Z. Xue, and Y. Zhang, "Bearing fault detection based on EMD and phase envelope analysis," Journal of Information \& Computational Science, vol. 5, no. 4, pp. 1781-1789, 2008. 

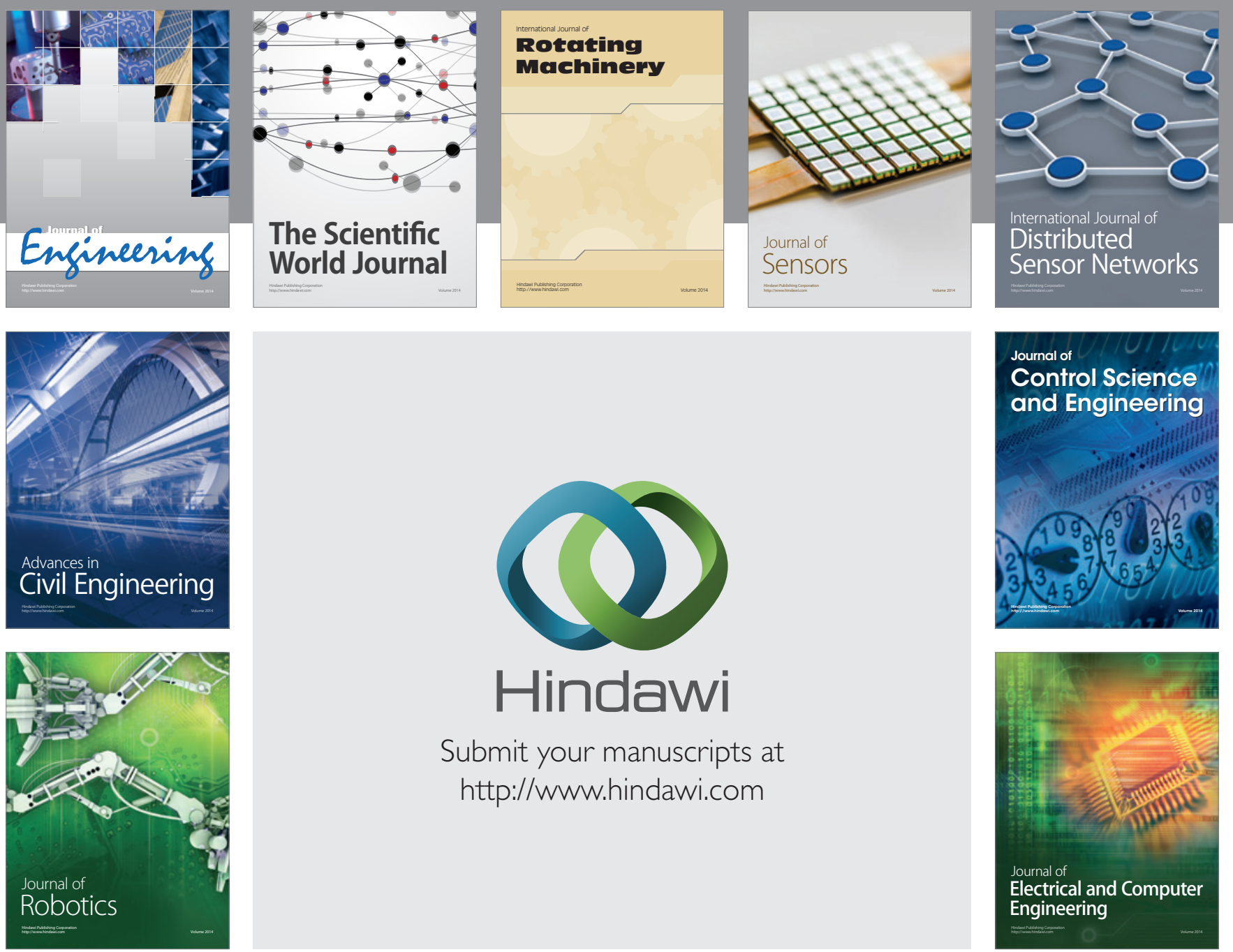

Submit your manuscripts at

http://www.hindawi.com
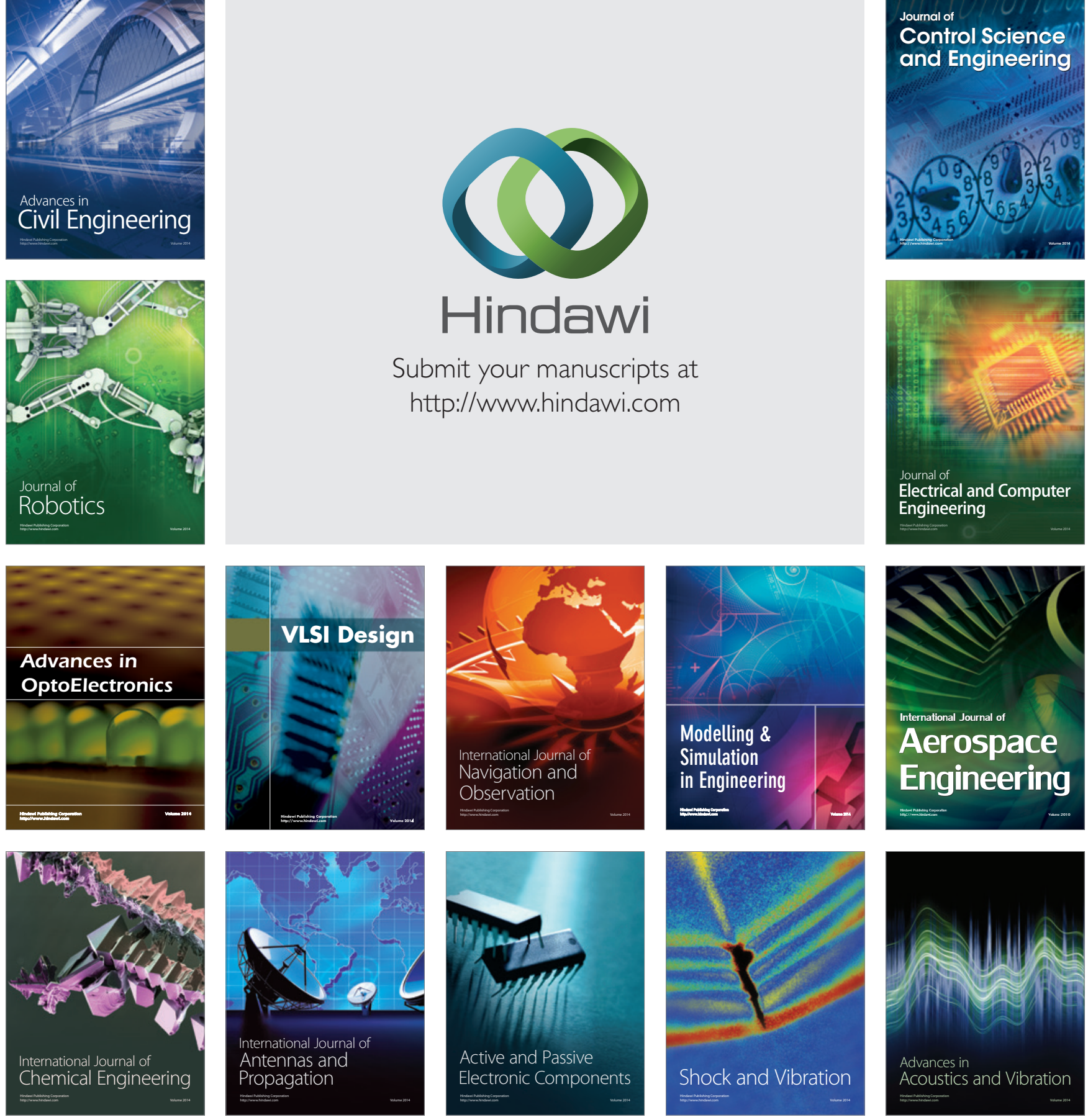